\title{
Local-scale faunal turnover on the deep Pacific seafloor
}

\author{
Craig R. McClain ${ }^{1, *}$, Jeffrey C. Nekola ${ }^{2}$, Linda Kuhnz ${ }^{3}$, James P. Barry ${ }^{3}$ \\ ${ }^{1}$ National Evolutionary Synthesis Center, 2024 W. Main Street, Durham, North Carolina 27705, USA \\ ${ }^{2}$ University of New Mexico, Department of Biology, Albuquerque, New Mexico 87131, USA \\ ${ }^{3}$ Monterey Bay Aquarium Research Institute, 7700 Sandholdt Rd., Moss Landing, Califorina 95039 USA
}

\begin{abstract}
The high biodiversity of the deep-sea floor is often attributed to high local coexistence of species achieved through microhabitat variation. Grassle \& Sanders (1973; Deep-Sea Res 34: 313-341) proposed that deep-sea species were differentially adapted to multiple and small-scale successional patches that varied across the landscape and through time. However, results from both manipulative experiments and precision sampling to test the patch-mosaic model of Grassle \& Sanders (1973) have varied, leading some authors to suggest that patch dynamics may be unimportant in explaining deep-sea biodiversity. We utilized a remotely operated vehicle and a rigid spatial sampling protocol to document macrofaunal turnover and individual species spatial dispersion at a $3203 \mathrm{~m}$ deep site in the Pacific Ocean over scales of 1 to $350 \mathrm{~m}$. We found high variability in assemblage composition and, in contrast to most previous work, we also found that intraspecific species aggregation was common. These findings suggest that patch dynamics and microhabitat variation are important in promoting local species coexistence in the deep-sea benthos.
\end{abstract}

KEY WORDS: Beta-diversity · Distance decay · Patch dynamics · Biodiversity • Patch mosaic • Turnover $\cdot$ Habitat heterogeneity

\section{INTRODUCTION}

At local scales in the deep-sea benthos, high coexistence of species is often linked to microhabitat variation. The creation of this spatial mosaic of patches is thought to reflect variability in sinking food parcels from surface waters, bioturbation from megafauna, or biogenic structures created by other organisms (Jumars 1975, Jumars \& Eckman 1983, Thistle 1983, Smith 1985, Jumars \& Wheatcroft 1989, McClain \& Barry 2010). Grassle \& Sanders (1973) envisioned carbon flux and bioturbation as small-scale disturbances producing successional sequences temporally out of phase and varying across the landscape. In their model, local coexistence is achieved through differential adaptation of species to successional age. However, disturbance models or temporal sequencing of successional changes need not be invoked. Food, biogenic structures, and bioturbation would produce spatial variation in environmental regimes, leading to different suites of species adapted for each regime. The patch-mosaic model remains the principal paradigm for explaining exceptional local diversity in the deep-sea benthos (reviewed in Etter \& Mullineaux 2001, Snelgrove \& Smith 2002), rivaling tropical rainforests and reaching 300 species within a square meter (Grassle 1989, Etter \& Mullineaux 2001).

This hypothesis inspired 2 lines of research — the use of precision sampling to detect local-scale faunal patchiness and manipulative experiments to create new patches - with neither providing definitive evidence for the role of patch dynamics (reviewed in Rex \& Etter 2010, p. 51-78). Frequently presented as evidence of high local species turnover, sampling often yields species accumulation curves that rarely express an asymptote (reviewed in Etter \& Mullineaux 2001, Snelgrove \& Smith 2002). Prior work has also focused on individual occurrences of species among $10 \times 10 \mathrm{~cm}$ subcores within $0.25 \mathrm{~m}^{2}$ box cores or $0.04 \mathrm{~m}^{2}$ Eckmanstyle cores taken with a remotely operated vehicle 
(ROV). Contrary to the expected species patchiness proposed under the patch-mosaic hypotheses, individual species are often no different than the null expectation of randomness, suggesting low response to environmental patchiness (Jumars 1975, 1976, 1978, Thistle 1978, Jumars \& Eckman 1983, Lamont et al. 1995, reviewed in Rex \& Etter 2010). As demonstrated in subsequent work, however, variance to mean ratios utilized by some of these studies can be ineffective as a measure of aggregation measured as departures from randomness (Hurlbert 1990). Whether the lack of aggregation found in previous work utilizing variance to mean ratios is an artifact demands further inquiry. Experimental work creating artificial mounds or enriching nutrients found assemblage shifts in only a small minority of species that are often opportunistic species not found in background assemblages (Smith et al. 1986, Snelgrove et al. 1992). This mixture of limited response led Rex \& Etter (2010, p. 77) to conclude that current evidence suggests patchy phenomena cannot account for high local species diversity.

Although small-scale turnover is the foremost hypothesis for explaining elevated deep-sea diversity, relatively few studies explicitly examine this idea and there is subsequently limited understanding of temporal and geographic variability in patchiness. Undoubtedly, this gap reflects the difficulty in precision sampling and experimental work in the remote deep sea (Rex \& Etter 2010, p. 51-78 and references within). Much work remains as it did when Jumars (1976) noted the lack of studies quantitatively relating faunal turnover to geographic location of and distances between samples. Rex \& Etter (2010) advocated 'much more precision sampling and experimental work needs to be done at local scales in deep-sea communities to better document patch structure and dynamics'.

Here, we document both macrofaunal turnover and individual species spatial dispersion at a deep-sea site (3203 m) in the eastern Pacific Ocean. We utilize an explicit spatial sampling protocol frequently used in plant community ecology that incorporates a nested and clustered sampling design. This design, combined with small scale precision sampling with a ROV, allowed us to compare patterns over spatial scales spanning from a few centimeters to $\sim 350 \mathrm{~m}$. We find enormous variability in assemblage composition at scales from a few centimeters to hundreds of meters that are likely related to variation in sedimentary carbon content. In contrast to previous work, we find that intraspecific species aggregation may be a common feature of the deep-sea floor across multiple spatial scales.

\section{MATERIALS AND METHODS}

In November 2006, we sampled a 3203 m deep site $\left(36.154098^{\circ} \mathrm{N}, 122.40852^{\circ} \mathrm{W}\right)$ with ROV 'Tiburon' of the Monterey Bay Aquarium Research Institute over the course of 3 dives (14 to 16 November 2006) (Fig. 1). The site was named Deadwood after a nearby woodfall experiment. We detected no substantial spatial variation in temperature, oxygen, and salinity at the site $\left(1.6^{\circ} \mathrm{C}, 3.0 \mathrm{ml} \mathrm{l}^{-1}\right.$, and $34.5 \mathrm{ppt}$ respectively).

We collected 44 sediment cores (inner core diameter: $7 \mathrm{~cm}$ ) with the ROV over a $350 \mathrm{~m}$ transect (Fig. 1). Bottom depth of samples varied less than $3 \mathrm{~m}$ (3201 to $3204 \mathrm{~m})$. At the 2 ends of the transect, we conducted a nested squares sampling by nesting 10 cores at the corners of $0.15 \mathrm{~m}, 0.5 \mathrm{~m}$, and $1 \mathrm{~m}$ sided squares (Fig. 1). This sampling design is commonly used in plant community ecology to effectively quantify spatial patterns (e.g. Palmer et al. 2007). In the sampling design, the cores are spatially non-independent, but it was our goal to document the degree of this non-independence in the observations. Every $50 \mathrm{~m}$ between the nested squares we sampled a cluster of 4 cores, for a total of 6 individual clusters between the 2 nested clusters (Fig. 1). The top 0 to $5 \mathrm{~cm}$ of each core was sieved for macrofauna through a $300 \mu \mathrm{m}$ mesh and preserved in $10 \%$ formalin. We also sieved the 5 to $10 \mathrm{~cm}$ fractions,

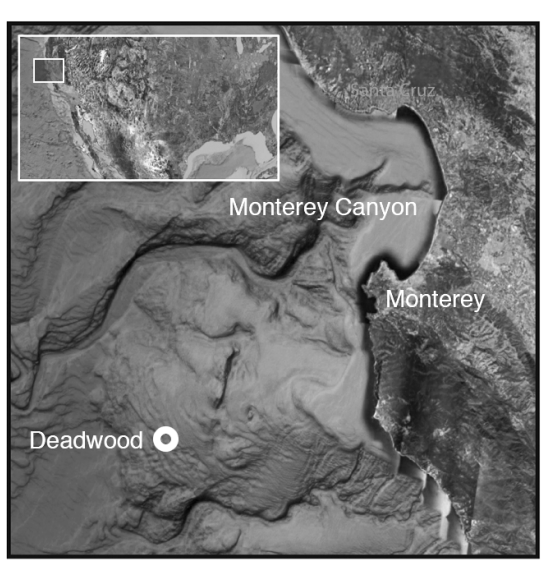

Fig. 1. Sampling locality Deadwood at $3203 \mathrm{~m}$ depth in the Northeastern Pacific Ocean off the Central California coast with an illustration of core sampling design at Deadwood over a 350 m transect. Different colour boxes along the $350 \mathrm{~m}$ transect indicate sections sampled during 3 separate dives. Open circles correspond to $7 \mathrm{~cm}$ diameter faunal cores taken with a remotely operated vehicle (ROV). Squares around the circles group core samples into clusters (numbered 1 to 8). Nested squares at each end of the transect indicate nested sampling of the 10 cores depicted at these sites (see Materials and methods). Sampling occurred over $3 \mathrm{~d}$ and $3 \mathrm{ROV}$ dives 
but they yielded no or few individuals and were not included in the analyses and discussions here. We identified all individuals to morphospecies and assigned them to known species when possible. Taxonomic experts were consulted for polychaetes and amphipods. At each $50 \mathrm{~m}$ cluster, an additional core was taken to quantify C:H:N composition and stable isotopes of carbon and nitrogen, which were measured on a Finnigin Mass Spectrometer in the laboratory of R. Dunbar (Stanford University).

We calculated species accumulation curves for macrofauna with Mao's Tau (Sobs) in EstimateS (see Fig. 2) (Colwell 2009). To examine how similarity among assemblages changes with distance along the transect, we used the distance decay of similarity method (Nekola \& White 1999). Because we were interested in changes in the decay of faunal composition, we quantified similarity between samples with Bray-Curtis Similarity on presence/absence data. All combinations of distance pairs and their similarities were plotted, and an exponential decay model, Similarity $=a \times \exp (b$ $\times$ Distance), was fitted to these relationships where $a$ represents the intercept and $b$ represents the slope of the log-linear relationship. Dissimilarity was calculated simply as 1 - Similarity. Halving-distance, over which a $50 \%$ change in composition occurs, was calculated by dividing $\ln 2$ by the slope of the exponential relationship.

Several multivariate analyses were conducted in PRIMER v6 (Clarke \& Gorley 2006). Bray-Curtis similarity was calculated between cores using both presence/absence and log-transformed abundance data, which allowed us to explore changes in both species composition and species dominance. Nonmetric multidimensional scaling (MDS) was used to visualize turnover between samples in a reduced multivariate space. MDSs were conducted with the standard 25 restarts and allowing equal dissimilarities to be represented in the final ordination by unequal distances. Using the RELATE test in PRIMER, which is analogous to a Mantel test, we correlated matrices of faunal similarity with distance between samples to test whether assemblage similarity is higher in closer cores. Distances between cores were calculated using the geographic coordinates. These geographic distances were used to populate the cells of the model matrix where the diagonal core, i.e. the distances between a core and itself, would be zero. This model matrix was correlated with the faunal similarity matrix, where a strong correlation would denote that geographic distance explains the faunal differences between cores.

We also modeled expected distance decay patterns when individuals were randomly assigned to quadrats while keeping total individual abundances per core identical to observed values. We implemented this model by randomizing individuals within a sample by species matrix such that row (species abundances) and column (core abundances) sums remained the same. We conducted 1000 iterations of the model. Ordinary least square regressions for pattern visualization and Mantel correlations for tests of statistical significance were calculated for each iteration in Matlab (code available from C. R. McClain).

To look at dispersion patterns of individual species, some previous deep-sea studies compared variance to mean ratios to a Poisson distribution of random spatial structure (Gage \& Geekie 1973, Jumars 1975, 1978, Thistle 1978). Later work revealed model populations all yielded variance to mean ratios of 1.0 corresponding to supposed randomness under a Poisson distribution, even when model populations were designed specifically to be aggregated (Hurlbert 1990). Thus, Hurlbert (1990) noted that this metric was 'useless as a measure of departure from randomness... [and] uninterpretable as measure of aggregation' and instead advocated for Morisita's index of aggregation (Morisita 1959).

We calculated Morisita's index as:

$$
\left[\mathrm{q} \sum \mathrm{n}_{i}\left(\mathrm{n}_{i}-1\right)\right] /[\mathrm{N}(\mathrm{N}-1)]
$$

where $\mathrm{q}$ is the number of samples at distance $(\mathrm{h})$ from each other, while $\mathrm{n}_{i}$ is the number of individuals in the $i$ th sample, and $\mathrm{N}$ is the total number of individuals in all samples. Morisita's index equals 1.0 when a population is randomly distributed, $>1.0$ if a population is clumped, and $<1.0$ if a population is regularly distributed. The specific value of Morisita's index 'indicates how many times more likely it is that 2 randomly chosen individuals of the same species will occur [...] compared with the expected probability for a random distribution of individuals' (White \& Gilchrist 2007, p. 1331). We calculated Morisita's index for adjacent cores (36 possible core comparisons), adjacent diagonal cores (16 comparisons), and distances rounded off to $0.25 \mathrm{~m}$ (4 comparisons), $0.50 \mathrm{~m}$ (18 comparisons), $0.75 \mathrm{~m}$ (14 comparisons), $1 \mathrm{~m}$ (26 comparisons), $1.25 \mathrm{~m}$ (6 comparisons), $1.5 \mathrm{~m}$ (4 comparisons), $50 \mathrm{~m}$ (96 comparisons), and $100 \mathrm{~m}$ (112 comparisons).

\section{RESULTS}

A total of 79 species and 1874 individuals were collected from the 44 cores. The mean number of individuals per core was 36.4 (range: 10 to 81) and the mean number of species per core was 8.8 (range: 5 to 15). Five of the species were represented by only 1 individual and 4 species by only 2 individuals among all cores. The species accumulation curve for Deadwood, although not asymptotic, does indicate that the addition of new species with increased sampling has slowed, 


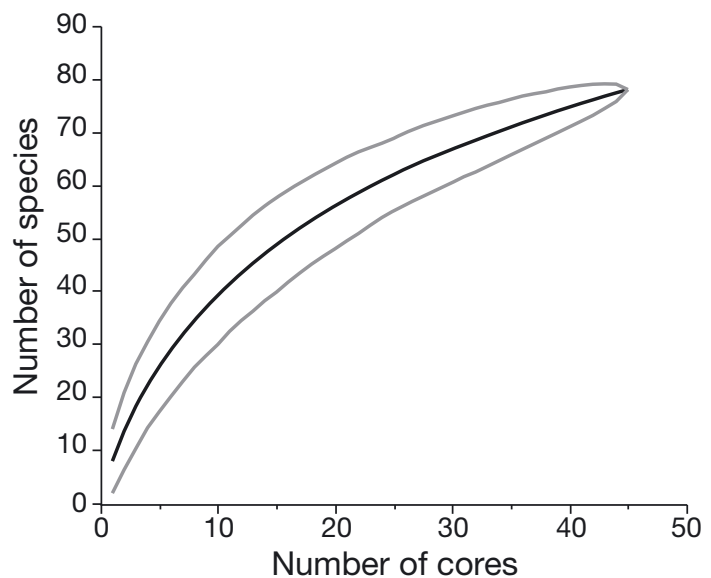

Fig. 2. Species accumulation curves for total macrofauna. Outer grey lines represent the $95 \%$ confidence intervals around the curve and that additional sampling would yield fewer new species (Fig. 2).

High variation in dissimilarity, i.e. 1 - Similarity, between cores occurred regardless of the geographic separation between them. The average compositional similarity based on the presence/absence data between adjacent cores was $88 \%$ (Fig. 3A). Macrofaunal communities were slightly more dissimilar at greater distances (Fig. 3A). However, compared to our model simulations, the presence of this weak relationship was statistically significant (Fig. 3B). The p-value was considerably lower and the correlation considerably higher than the vast majority (95\%) of the simulations (Fig. 3B-D). The halving distance, at which a $50 \%$ change in compositional similarity occurs, was $7.5 \mathrm{~km}$ (or not to extrapolate beyond our dataset: $\sim 1.5 \%$ for every $200 \mathrm{~m}$ ).
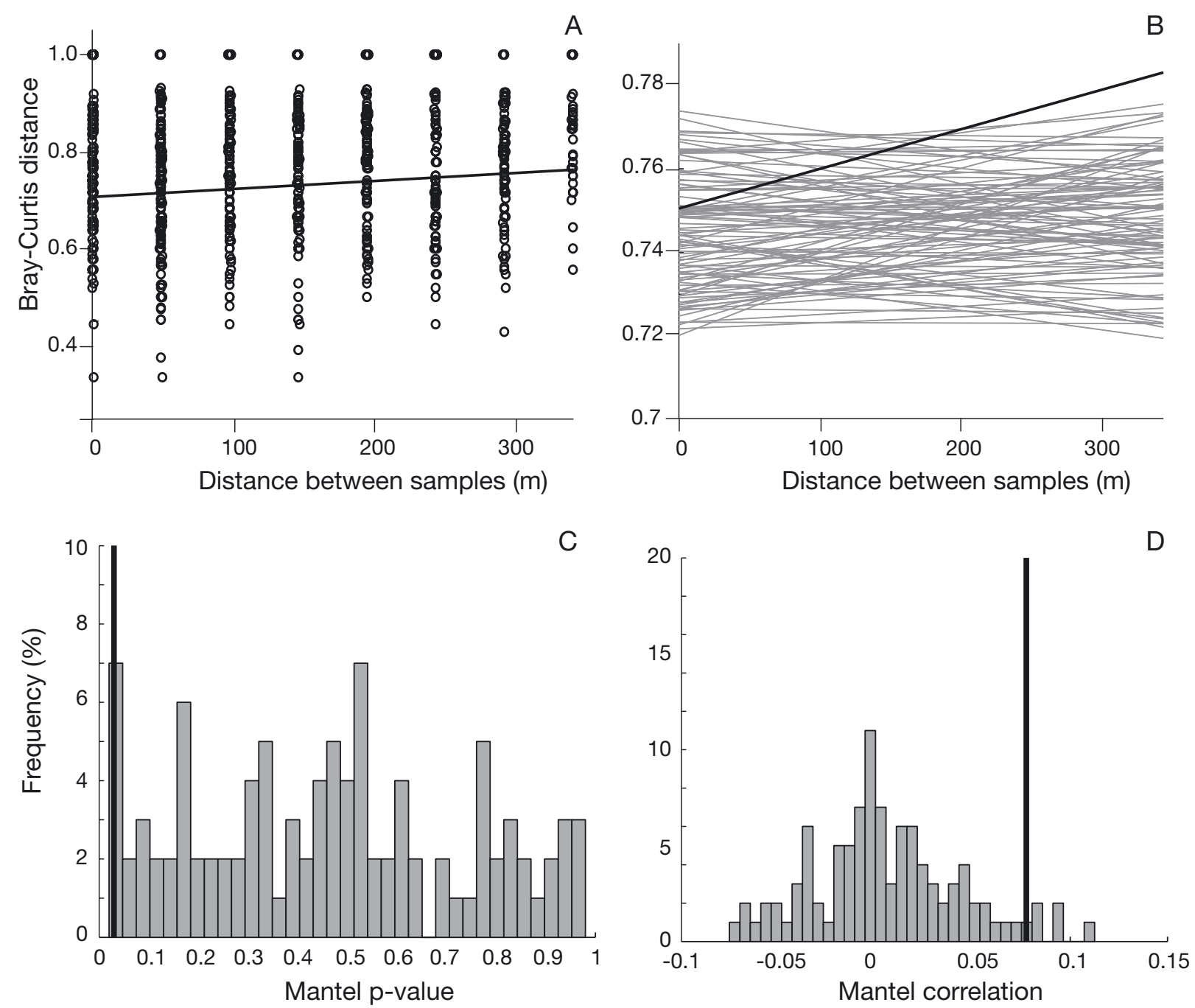

Fig. 3. Distance decay relationships for macrofauna. (A) Distance decay relationship with empirical points and fitted relationship given as Similarity $=0.25 \times \exp ($ Distance $\times-0.00009)$. Solid black line is the exponential decay fit such that Similarity $=a \times \exp ($ Distance $\times b$ ). (B) Fitted distance decay relationship, with Bray-Curtis distance (solid black line) and simulations (gray lines). Frequency distributions for simulations of (C) Mantel p-values and (D) Mantel correlations compared to empirical patterns (solid black lines) 
MDS plots based on presence/absence or logtransformed abundances showed a weak spatial signal among individual cores, with increasing distance associated with increasing dissimilarity (Fig. 4A,B). But the MDS should be interpreted cautiously since stress levels were high. Stresses in excess of 0.20 indicate a complex multivariate structure for assemblage similarity that is not easily reconciled into 2 dimensions. This interpretation is consistent with our other findings of variation in compositional dissimilarity irrespective of geographic separation (Fig. 3A). The correlations between the similarity matrix based on the assemblage
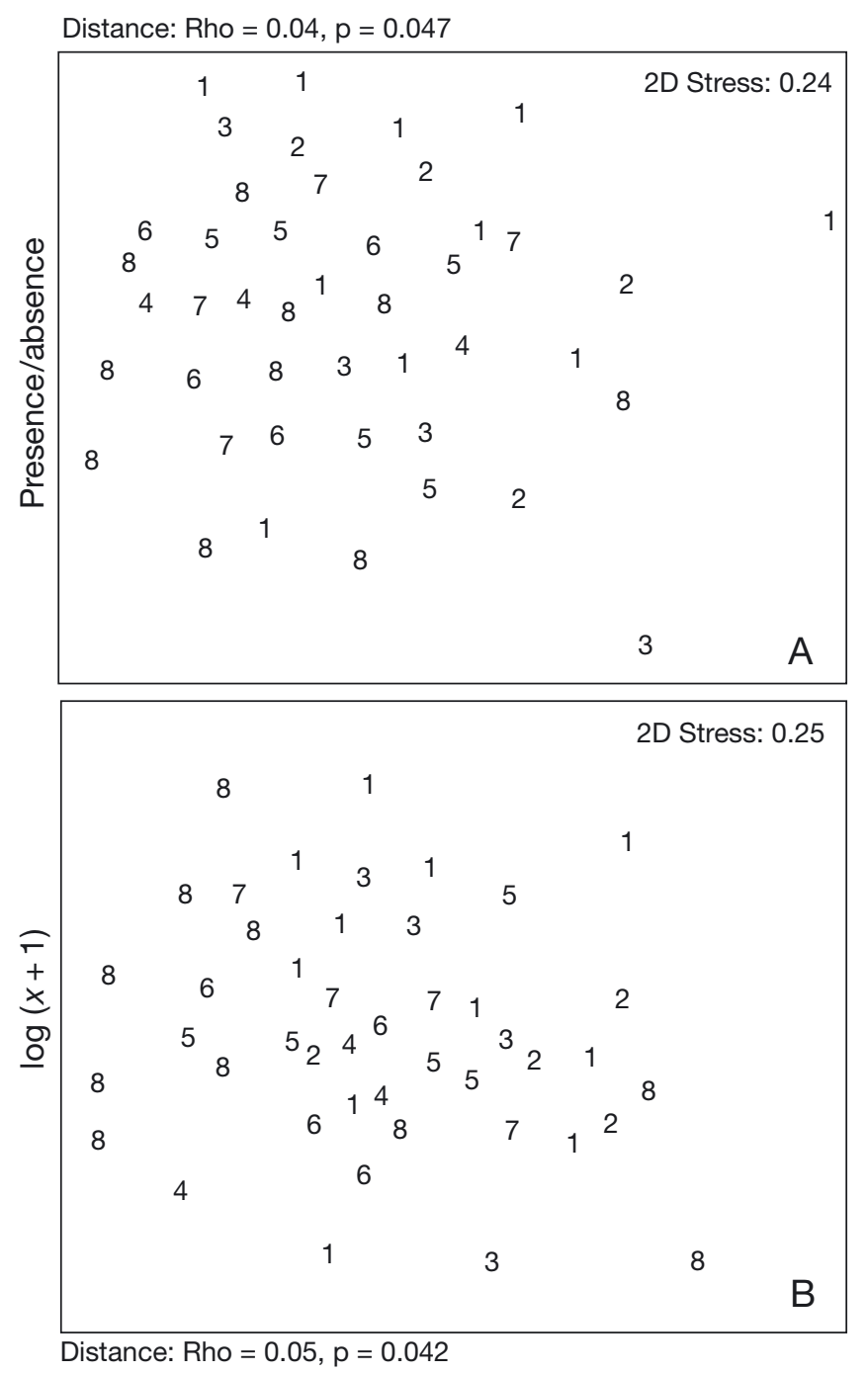

data and a geographic distance matrix are low but significant $(\mathrm{Rho}=0.04, \mathrm{p}=0.047$, and Rho $=0.05, \mathrm{p}=$ 0.042 respectively), suggesting loose spatial structure in the data. Among quadrats of cores at every $50 \mathrm{~m}$ (Fig. 1) a weak and insignificant correlation of increasing distance with increasing dissimilarity existed (Rho $=0.05, \mathrm{p}=0.094$, and Rho $=0.06, \mathrm{p}=0.078$ respectively) (Fig. 4A,B). The correlation among faunal similarity and percent sediment carbon is much stronger for both presence/absence data $(\mathrm{Rho}=0.42, \mathrm{p}=0.021$ ) and log-transformed abundance data (Rho $=0.31, \mathrm{p}=$ 0.036). No correlation existed between the abundance

Distance: $\mathrm{Rho}=0.05, \mathrm{p}=0.094$

$\%$ Carbon: $\mathrm{Rho}=0.42, \mathrm{p}=0.021$
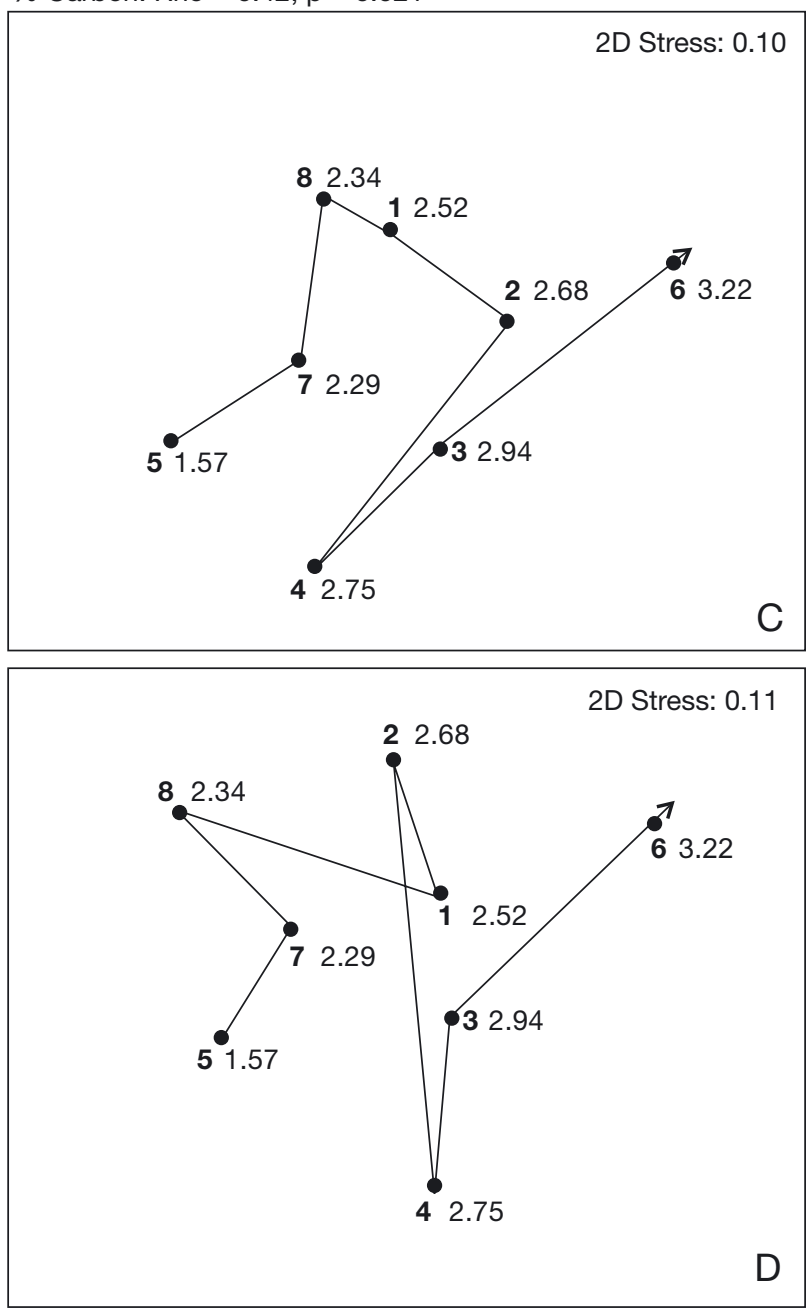

Distance: $\mathrm{Rho}=0.06, \mathrm{p}=0.078$

$\%$ Carbon: Rho $=0.31, p=0.036$

Fig. 4. Nonmetric multidimensional scaling (MDS) based on Bray-Curtis similarity of (A) presence/absence and (B) logtransformed data for individual cores. Numbers indicate the cluster in which the core occurred (see Fig. 1). MDS on Bray-Curtis similarity of (C) presence/absence and (D) log-transformed data for clusters of 4 adjacent cores taken every $50 \mathrm{~m}$ (6 individual clusters and 2 nested clusters). Numbers near points indicate the cluster number (see Fig. 1) and percent carbon. Lines connect clusters from the lowest to highest percent carbon. Rho and p-values are given for the correlation between the empirical similarity matrix and a model matrix based on geographic distance between cores as determined by the RELATE routine, similar to a Mantel test, in PRIMER. For $50 \mathrm{~m}$ clusters, Rho and p-values are also given for the correlation between the empirical similarity matrix and a model matrix based on similarity in percent carbon 

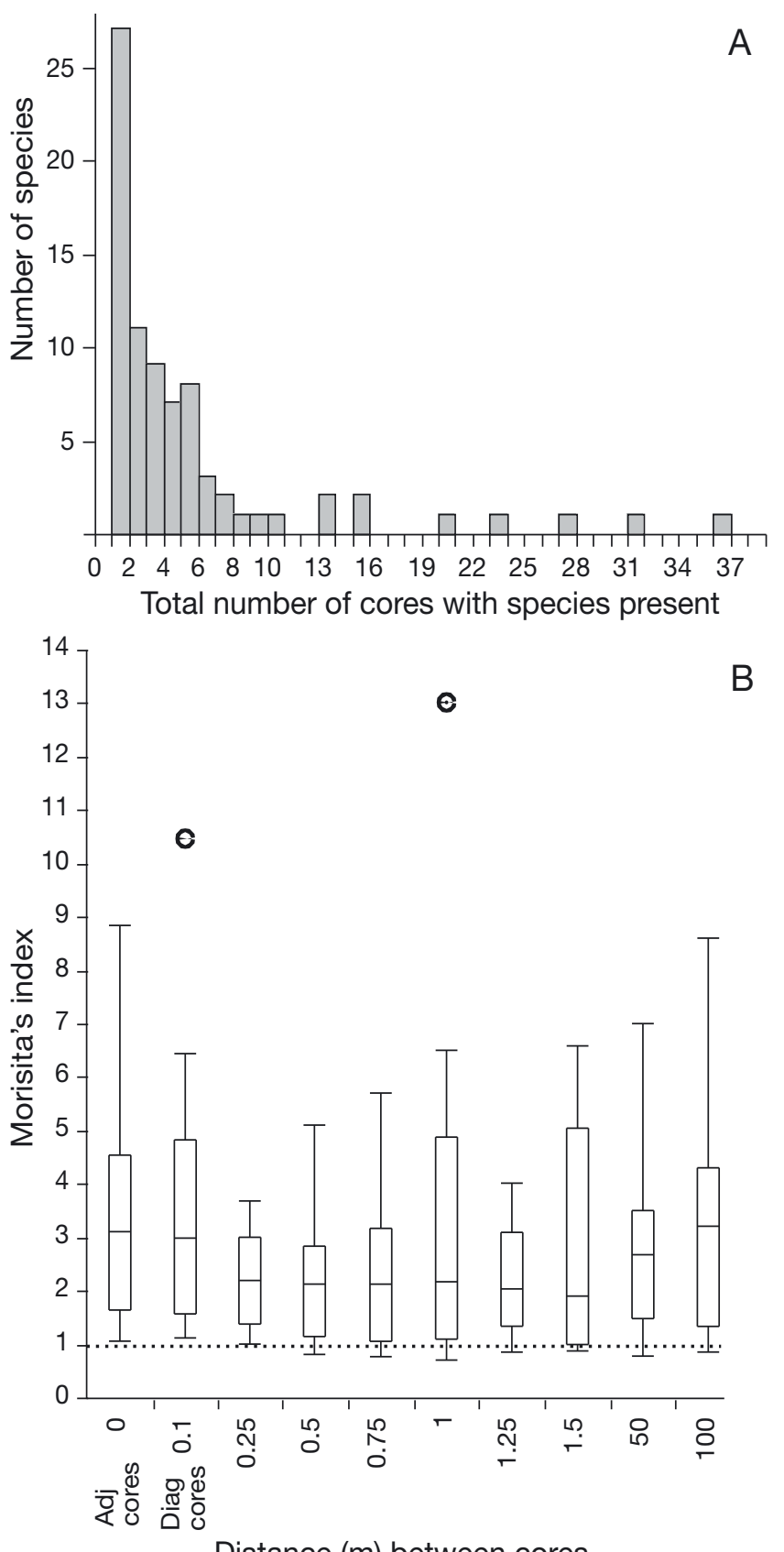

Distance $(m)$ between cores

Fig. 5. (A) Number of species that occur in $\mathrm{n}$ cores where $\mathrm{n}=1$ to 44 . (B) Distribution of Morisita's Index of spatial aggregation among the most abundant 20 species versus varying spatial distances in the present study. Morisita's index equals 1.0 (dashed line) when a population is randomly distributed, $>1.0$ if a population is clumped, and $<1.0$ if a population is regularly distributed. Upper and lower bounds: 25th and 75th quartiles; Line in the box: median; Whiskers: lowest datum still within 1.5 IQR of the lower quartile and the highest datum still within 1.5 IQR of the upper quartile. The outlying points ( ) at 0.1 and $1 \mathrm{~m}$ distance between cores represent the bivalve Tindaria compressa and the polychaete Prionospio delta respectively. Adjacent (adj.) and diagonal (diag) cores refer to pairs within the same cluster of individuals within quadrats of cores and percent sediment carbon $(p=0.5478)$. This implies compositional differences are unrelated to differences related to variation in abundance.

Visualizing the spatial dispersion among 10 of the most common species showed considerable patchiness among samples, even at the interspecific level (Fig. S1 in the supplement at www.int-res.com/articles/suppl/ m422p193_supp.pdf). A total of 20 species were abundant enough and occurred in more than 1 core cluster to analyze aggregation (Fig. 5), while 27 species occurred in only 1 core (Fig. 5A). All of the 20 species used for aggregation analysis showed significant aggregation. On average, species were 2.5 times more likely than expected by chance to encounter an individual of the same species in another core, whether separated by a few centimeters or several meters. There was a tendency, although nonsignificant, for Morisita's Index to be higher among adjacent and diagonal cores and at distances greater than $50 \mathrm{~m}$ (Fig. 5). Differences in aggregation among species of major clades, e.g. Polychaeta, Bivalvia, Aplacophora, Isopoda, and Tanaidacea, were not detectable (data not shown).

\section{DISCUSSION}

Using precision sampling with an ROV, we quantified a high degree of variability in faunal composition at small spatial scales on a backdrop of a weak but significant pattern of species turnover across a $350 \mathrm{~m}$ transect along the deep-sea bottom. In general, 2 samples taken adjacent to one another were only slightly more likely to be compositionally similar than those far apart, as demonstrated by the large scatter in both the distance decay and MDS plots (Figs. 3 \& 4). Adjacent or $350 \mathrm{~m}$ distant samples on average shared only $22 \%$ of their species in common but in some cases reached levels of $\sim 70 \%$ compositional similarity. Despite this considerable variation in species makeup, we did find a significant, though small, distance decay relationship such that distant assemblages exhibited slightly less similarity than proximate ones. Across the Deadwood site, $200 \mathrm{~m}$ distance yielded a $1.5 \%$ change in fauna. This percent change was much greater than that encountered in our simulations of random assemblages indicating that the ecological structure of the deep-sea floor is not entirely stochastic.

Nonrandom spatial structure was also exhibited within the 20 most abundant species across the spatial scales we studied. Our findings contrast to those from shallower but reasonably nearby sites in the Pacific ( 1200 m deep) and distant sites in the Atlantic (2875 m deep) demonstrating that species dispersion patterns 
at small spatial scales on the seabed are rarely different from random patterns (Jumars 1975, 1976, 1978, Gage 1977, Thistle 1978, Lamont et al. 1995). At intermediate scales $(\sim 100$ to $200 \mathrm{~m})$, prior studies at shallower upper bathyal and deeper abyssal depths also found few species departing from a random dispersion (Jumars 1975, 1976, 1978, Gage 1977, Thistle 1978, Lamont et al. 1995, Cosson et al. 1997). However, at lower bathyal depths, like those in the present study, aggregation within species is frequent (Lamont et al. 1995, Cosson et al. 1997, reviewed in Rex \& Etter 2010, p. 51-78). Some of these differences in findings likely reflect methodological differences, in particular our choice of Morisita's Index over variance to mean ratios. The latter metric is unlikely to detect departures from randomness even when they exist (Hurlbert 1990).

We propose that increased intraspecific aggregation along with the decrease in compositional similarity with distance among assemblages may reflect a more heterogeneous environment typical of upper bathyal habitats. Mid-depth regions represent considerable heterogeneity in carbon flux, topography, sediments, and currents at large spatial scales (Carney 2005, Zardus et al. 2006). As such, species of the upper bathyal often exhibit smaller geographic ranges (Wilson \& Kaufmann 1987, Allen \& Sanders 1996) and greater genetic divergence (Etter \& Rex 1990, Zardus et al. 2006). How habitat heterogeneity varies bathymetrically at the local scales relevant to this study remains virtually unexplored. Etter \& Grassle (1992) demonstrated that greater sediment heterogeneity within $0.25 \mathrm{~m}^{2}$ boxcores correlated with greater alphadiversity among macrofauna in the Northwest Atlantic. Although no such study exists for the Pacific for direct comparison, the greatest sediment heterogeneity occurred at $\sim 1250 \mathrm{~m}$ depth, much shallower than the lower bathyal depths discussed here. Greater competition, as reflected by character displacement, may also occur among species at intermediate depths (McClain 2005). This pattern contrasts the abyssal plains, where stochastic variation in recruitment combined with low population densities (Rex et al. 2005) may generate assemblages more consistent with neutral expectations (Hubbell 2001).

A spatial mosaic of patches on the deep-sea floor, whether resulting from disturbance or the presence of biogenic structures, remains one of the principal explanations for high alpha-diversity of the deep-sea benthos (Grassle \& Sanders 1973, Jumars 1975, reviewed in Rex \& Etter 2010, p. 51-78). Recent work demonstrates that disturbance from bioturbation and gradients in carbon flux can also work in concert to increase beta-diversity over tens of meters (McClain \& Barry 2010). This assortment of patches may persist for many years as a result of low colonization, bioturba- tion, and benthic disturbance rates (Etter \& Mullineaux 2001). Feeding depressions, fecal pellets, mounds, tubes, tracks, and tests create physical heterogeneity on the seafloor (Thistle 1979, 1983, Jumars \& Eckman 1983, Etter \& Mullineaux 2001, Snelgrove \& Smith 2002). Bioturbation by megafauna may create patches varying in disturbance regimes (Jumars \& Eckman 1983, Smith et al. 2008). Video from the ROV during the 3 dives reported here revealed substantial microtopography from mounds, depressions, and feeding tracks. Deposit-feeding echinoids, holothurians, and ophiuriods were also seen.

Carbon and sediment are also heterogeneously distributed at small scales across the deep-sea floor (Smith 1985, Jumars \& Wheatcroft 1989, Etter \& Grassle 1992, Gooday 2002). Discarded larvacean mucus nets and other detrital aggregations produce patches of varying food quality and quantity (Alldredge \& Silver 1988, Robison et al. 2005). Detrital flux can amass in local depressions leading to food-rich patches (Jumars \& Eckman 1983). We detect a significant correlation between compositional and structural variation in assemblages at $50 \mathrm{~m}$ and percent carbon of the sediment. Although more research will be required linking beta-diversity to carbon heterogeneity across spatial scales, our work suggests that deep-sea faunal patchiness is partly related to spatial patchiness in food.

Our results highlight the potential scales of ecological variability on the deep-sea floor. The weak but significant distance decay relationship of more distant assemblages exhibiting less similarity than proximate ones suggests that even at small scales a variety of ecological processes such as dispersal dynamics, local rarity, reproductive aggregation, patch extinction, and competitive exclusion may contribute to significant species patterning. We also show that local species coexistence may be high because of extremely patchy distributions of individual species, potentially reflecting small-scale environmental heterogeneity. Heterogeneity in carbon flux to the deep-sea floor is well known, and here we begin to explicitly tie this variable to local-scale beta-diversity in the deep sea. More work is obviously needed to quantify environmental and faunal heterogeneity at local scales, as well as possible geographic variation in these relationships. Here, we demonstrate that distribution of assemblages and species is not stochastic and that patchiness may explain the exceptional local diversity in the deep-sea benthos.

Acknowledgements. This work was supported by the Monterey Bay Aquarium Research Institute (projects 200002, 900703, 900608), and the David and Lucile Packard Foundation. C.R.M. was supported by a Monterey Bay Aquarium Research Institute Postdoctoral Fellowship and the National Evolutionary Synthesis Center. We also thank the pilots of the ROV 'Tiburon' and 
the crew of the RV 'Western Flyer'. We are grateful for the assistance of C. Leong, K. Buck, C. Lovera, P. Slattery, and P. Whaling. M. Rex provided valuable input on a prior version of this manuscript. M. Gaither-McClain provided editorial assistance and loving patience with the first author.

\section{LITERATURE CITED}

Alldredge AL, Silver M (1988) Characteristics, dynamics and significance of marine snow. Prog Oceanogr 20:41-82

Allen JA, Sanders HL (1996) The zoogeography, diversity and origin of the deep-sea protobranch bivalves of the Atlantic: the epilogue. Prog Oceanogr 38:95-153

Carney RS (2005) Zonation of deep biota on continental margins. Oceanogr Mar Biol Annu Rev 43:211-278

Clarke KR, Gorley RN (2006) Primer. Primer-E, Plymouth

Colwell RK (2009) EstimateS: statistical estimation of species richness and shared species from samples. Version 8. Available at: http://viceroy.eeb.uconn.edu/estimates

> Cosson N, Sibuet M, Galeron J (1997) Community structure and spatial heterogeneity of the deep-sea macrofauna at three contrasting stations in the tropical Northeast Atlantic. Deep-Sea Res I 44:247-269

Etter RJ, Grassle JF (1992) Patterns of species diversity in the deep sea as a function of sediment particle size diversity. Nature 360:576-578

Etter RJ, Mullineaux LS (2001) Deep-sea communities. In: Bertness MD, Gaines SD, Hay ME (eds) Marine community ecology. Sinauer Associates, Inc., Sunderland, MA, p 367-394

Etter RJ, Rex MA (1990) Population differentiation decreases with depth in deep-sea gastropods. Deep-Sea Res 37: $1251-1261$

Gage JD (1977) Structure of the abyssal macrobenthic community in the Rockall Trough. European Symposium on Marine Biology 11:247-260

Gage JD, Geekie AD (1973) Community structure of the benthos in the Scottish Sea-lochs. II. Spatial pattern. Mar Biol 19:41-53

> Gooday AJ (2002) Biological responses to seasonally varying fluxes of organic matter to the ocean floor: a review. J Oceanogr 58:305-332

$>$ Grassle JF (1989) Species diversity in deep-sea communities. Trends Ecol Evol 4:12-15

Grassle JF, Sanders HL (1973) Life histories and the role of disturbance. Deep-Sea Res 34:313-341

Hubbell SP (2001) The unified theory of biodiversity and biogeography. Princeton University Press, Princeton, NJ

Hurlbert SH (1990) Spatial distribution of the montane unicorn. Oikos 58:257-271

Jumars PA (1975) Environmental grain and polychaete species' diversity in a bathyal community. Mar Biol 30:253-266

Jumars PA (1976) Deep-sea species diversity: Does it have a characteristic scale? J Mar Res 34:217-246

Jumars PA (1978) Spatial autocorrelation with RUM: vertical and horizontal structure of a bathyal benthic community. Deep-Sea Res 25:589-604

Jumars PA, Eckman J (1983) Spatial structure within deepsea benthic communities. In: Rowe GT (ed) The Sea, Vol 8. Wily-Interscience, New York, NY, p 399-451

Jumars PA, Wheatcroft RA (1989) Responses of benthos to changing food quality and quantity, with a focus on deposit feeding and bioturbation. In: Berger WH, Smetacek VS, Wefer G (eds) Productivity of the ocean: past and present. Wiley, New York, NY, p 235-253
Lamont PA, Gage JD, Tyler PA (1995) Deep-sea macrobenthic communities at contrasting sites off Portugal, preliminary results: II Spatial disperson. Int Rev Gesamten Hydrobiol Hydrograph 80:251-265

> McClain CR (2005) Bathymetric patterns of morphological disparity in deep-sea gastropods from the western North Atlantic Basin. Evolution 59:1492-1499

McClain CR, Barry J (2010) Habitat heterogeneity, biogenic disturbance, and resource availability work in concert to regulate biodiversity in deep submarine canyons. Ecology 91:964-976

Morisita M (1959) Measuring the dispersion of individuals and analysis of the distributional patterns. Memoirs of the Faculty of Science Kyushu University Series E 2:215-235

> Nekola JC, White PS (1999) The distance decay of similarity in biogeography and ecology. J Biogeogr 26:867-878

> Palmer MW, Peet RK, Reed RA, Xi W, White PS (2007) A multiscale study of vascular plants in a North Carolina piedmont forest. Ecology 88:2674

Rex MA, Etter RJ (2010) Deep-sea biodiversity: pattern and scale. Harvard University Press, Cambridge, MA

$>$ Rex MA, McClain CR, Johnson NA, Etter RJ, Allen JA, Bouchet P, Waren A (2005) A source-sink hypothesis for abyssal biodiversity. Am Nat 165:163-178

Robison BH, Reisenbichler KR, Sherlock RE (2005) Giant larvacean houses: rapid carbon transport to the deep sea floor. Science 308:1609-1611

Smith CR (1985) Food for the deep sea: utilization, dispersal, and flux of nekton falls at the Santa Catalina Basin floor. Deep-Sea Res 32:417-422

Smith CR, Jumars PA, Demaster DJ (1986) In situ studies of megafaunal mounds indicate rapid sediment turnover and community response at the deep-sea floor. Nature 323: 251-253

Smith CR, De Leo FC, Bernardino AF, Sweetman AK, Arbizu PM (2008) Abyssal food limitation, ecosystem structure and climate change. Trends Ecol Evol 23:518-528

Snelgrove PV, Smith CR (2002) A riot of species in an environmental calm: the paradox of the species-rich deep-sea floor. Oceanogr Mar Biol Annu Rev 40:311-342

Snelgrove PVR, Grassle JF, Petrecca RF (1992) The role of food patches in maintaining high deep-sea diversity: field experiments with hydrodynamically unbiased colonization trays. Limnol Oceanogr 37:1543-1550

Thistle D (1978) Harpacticoid dispersion patterns: implications for deep-sea diversity maintenance. J Mar Res 36: 377-397

Thistle D (1979) Deep-sea harpacticoid copepod diversity maintenance: the role of polychaetes. Mar Biol 52:371-376

Thistle D (1983) The role of biologically produced habitat heterogeneity in deep-sea diversity maintenance. Deep-Sea Res 30:1235-1245

White EP, Gilchrist M (2007) Effects of population-level aggregation, autocorrelation, and interspecific association on the species-time relationship in two desert communities. Evol Ecol Res 9:1329-1347

Wilson RR, Kaufmann RS (1987) Seamount biota and biogeography. In: Keating BH, Fryer P, Batiza R, Boehlert GW (eds) Seamounts, islands, and atolls. Geophysical Monograph 43. American Geophysical Union, Washington, DC, p 227-237

Zardus JD, Etter RJ, Chase MR, Rex MA, Boyle EE (2006) Bathymetric and geographic population structure in the pan-Atlantic deep-sea bivalve Deminucula atacellana (Schenck, 1939). Mol Ecol 15:639-651

Submitted: April 2, 2010; Accepted: November 4, 2010

Proofs received from author(s): January 17, 2011 